\title{
Efeito da amônia sobre a fertilização artificial de ovócitos e no desenvolvimento inicial do jundiá cinza (Rhamdia quelen; Siluriformes, Heptapteridae)
}

\section{Effect of ammonia on the artificial fertilization of oocytes and early development of jundia (Rhamdia quelen, Siluriformes, Heptapteridae)}

\author{
Eléxio Vidal ${ }^{1 *}$; Denise Nascimento de Bastos ${ }^{1}$; Edna Aparecida Oliveira ${ }^{1}$; \\ Arno Juliano Butzge ${ }^{2}$; Juliana Kasper Mewes²; Gilmar Baumgartner'; \\ Paulo Vanderlei Sanches ${ }^{1}$; Robie Allan Bombardelli ${ }^{1}$
}

\begin{abstract}
Resumo
O presente estudo teve como objetivo avaliar os efeitos de diferentes níveis de amônia, sobre os parâmetros de motilidade espermática, a fertilização artificial e o desenvolvimento inicial do jundiá cinza (Rhamdia quelen; Siluriformes, Heptapteridae). O sêmen foi ativado utilizando a diluição de 1:70 (sêmen:água) e avaliado pelo método CASA. Foi considerado como tratamento água contendo 0,$06 ; 0,28 ; 0,50 ; 0,77$ e $0,84 \mathrm{mg}$ de amônia total.L de água ${ }^{-1}$, tanto para ativação espermática quanto para fertilização e incubação dos ovócitos. A incubação era composta por um sistema de recirculação de $250 \mathrm{~L}$ para cada tratamento, com quatro incubadoras, sendo que cada incubadora tinha volume útil de 2,5L com aproximadamente 3.800 ovos. Foram avaliados os parâmetros de motilidade espermática, de taxa de fertilização, unidade térmica acumulada para início e término da eclosão, taxa de eclosão, desenvolvimento embrionário e percentual de larvas normais. Os resultados foram submetidos a análise de variância e a análise de regressão a um nível de $5 \%$ de probabilidade. Os níveis de amônia presentes na água não interferiram sobre os parâmetros avaliados ( $\mathrm{p}>0,05)$, exceto quanto ao desenvolvimento embrionário inicial, onde os embriões expostos aos maiores níveis de contaminação por amônia apresentaram maiores $(\mathrm{p}<0,05)$ taxas de fechamento do blastóporo. De modo geral, a presença de até $0,84 \mathrm{mg}$ de amônia total.L de água ${ }^{-1}$ acelera o desenvolvimento inicial embrionário mas não interferem nas taxas de fertilização e eclosão.
\end{abstract}

Palavras-chave: Amônia, embrião, larvas, peixe, poluente

\begin{abstract}
The aim study it had as objective to evaluate the effect of different ammonia levels, on the parameters of sperm motility, the artificial fertilization and the early development of jundia (Rhamdia quelen; Siluriformes, Heptapteridae). The semen was activated using the dilution of 1:70 (semen:water) and evaluated by the CASA method. It was considered as treatment water contending 0,$06 ; 0,28 ; 0,50 ; 0,77$
\end{abstract}

\footnotetext{
${ }^{1}$ Programa de Pós Graduação em Recursos Pesqueiros e Engenharia de Pesca, Universidade Estadual do Oeste do Paraná, UNIOESTE, Toledo, PR.E-mail: elexiovidal@yahoo.com.br; denisenbastos@hotmail.com; edna.aparecida.oliveira@gmail.com; gilmar_baum@yahoo.com.br; pvsanches@yahoo.com.br; rabombardelli@gmail.com

2 Biólogos da Pontifícia Universidade Católica do Paraná PUCPR, Toledo, PR. E-mail: juliano.butzge@hotmail.com; julimewes@ hotmail.com

* Autor para correspondência
} 
and $0,84 \mathrm{mg}$ of total ammonia.water ${ }^{-1} \mathrm{~L}$, so for sperm activation as for fertilization and incubation of the oocytes. The incubation was composed for a recirculation system of $250 \mathrm{~L}$ for each treatment, with four incubators, being that each incubator approximately had useful volume of 2,5L with 3,800 eggs. Had been evaluated the parameters of sperm motility, fertilization rate, thermal unit accumulated for beginning and ending of the hatching, hatching rate, embryonic and percentile development of normal larvae. The results had been submitted the analysis of variance and the analysis of regression to a $5 \%$ of probability level. The ammonia levels gifts in the water did not interfere on the evaluated parameters ( $>0,05$ ), except about to the early embryonic development, where the embryos displayed to the biggest levels of contamination for ammonia had presented greaters $(p>0,05)$ closing of the blastopore rates. In general way, the presence of until $0,84 \mathrm{mg}$ of total ammonia. Water-1 L speeds up the embryonic early development but they do not interfere with the fertilization and hatching rates.

Key words: Ammonia, embryo, larvae, fish, pollutant

\section{Introdução}

A exploração comercial de peixes vem se expandindo muito nos últimos anos, segundo a FAO (2010) a produção mundial na aquicultura em 2009 foi de 55,1 milhões de toneladas, dos quais $54,7 \%$ representam o cultivo de peixes de água doce. No Brasil, inúmeras espécies nativas de peixes apresentam potencial de serem exploradas pela piscicultura.

De acordo com Maffezzolli e Nuñer (2006), uma espécie que desperta grande interesse para a piscicultura da região sul do Brasil, por seu crescimento acelerado, inclusive nos meses mais frios, é o jundiá, Rhamdia quelen (QUOY; GAIMARD, 1824). A produção desta espécie entre 2007 e 2009, correspondeu a 1089 toneladas (MPA, 2009). Esta espécie possui importância não só econômica, mas também ecológica (MIRON et al., 2008) em função de uma estratégia alimentar generalista (KÜTTER; BEMVENUTI; MORESCO, 2009) e pela sua ampla distribuição geográfica, sendo encontrada do México até a Argentina (PERDICES et al., 2002).

$R$. quelen assumiu seu status produtivo devido à características relacionadas ao relativo domínio da tecnologia de sua criação (BOMBARDELLI et al., 2006). Contudo, alguns aspectos referentes à reprodução artificial destes peixes ainda são pouco compreendidos, a exemplo dos fatores que influenciam na fertilização artificial dos oócitos e o desenvolvimento inicial dos embriões e das proles, que podem depender da nutrição dos reprodutores (TESSARO et al., 2012). A influência de fatores como a temperatura e presença de metais pesados como o chumbo e o cádmio sobre estes parâmetros tem sido discutida em estudos como Hilbig et al. (2008), Sanches et al. (2011) e Witeck et al. (2011).

A amônia presente na água pode ser um fator limitante ao desenvolvimento e até para a sobrevivência de peixes (WANG; WALSH, 2000), seja em ambiente artificial ou natural. A origem de compostos nitrogenados na água pode ser de efluente da própria aquicultura (BALDISSEROTTO; RADUNZ NETO, 2004), proveniente da alimentação e/ou excreção dos peixes (PEREIRA; MERCANTE, 2005). A amônia na forma não ionizada $\left(\mathrm{NH}_{3}\right)$ pode ser absorvida pelo epitélio branquial (SILVA; JARDIM, 2006) e promover alterações fisiológicas como alteração do pH sanguíneo (BECKER et al., 2009), danos neurais (RAABE; LIN, 1985) e até morte (RANDALL; TSUI, 2002).

Em geral, as informações a respeito dos níveis de tolerância a amônia pelos peixes juvenis ou adultos são abundantes na literatura, visto a importância deste tema para prevenir as perdas produtivas em sistemas de criação. Apesar de serem estabelecidos os limites aceitáveis para peixes juvenis e adultos entre 0,15 a $1,00 \mathrm{mg}$ de amônia/L de água (OSTRENSKY; BOEGER, 1998), muito pouca informação esta disponível sobre os efeitos da amônia e sobre os seus limites mínimos e máximos 
toleráveis para peixes nas fases embrionárias ou larvais, bem como seus efeitos sobre a ativação dos gametas.

Neste sentido, este estudo teve como objetivo avaliar os efeitos de diferentes níveis de amônia total sobre os parâmetros espermáticos, fertilização e eclosão de ovos e o desenvolvimento embrionário do $R$. quelen.

\section{Material e Métodos}

Os efeitos das diferentes concentrações de amônia na água sobre os gametas, embriões e larvas do Rhamdia quelen foram testados no período de outubro a novembro de 2010, utilizado-se quatro machos e duas fêmeas de $R$. quelen. Para tanto, diferentes quantidades de hidróxido de amônia $\left(\mathrm{NH}_{3} \mathrm{OH}\right)$ foram diluídas na água até atingir as concentrações correspondentes a 0,$06 ; 0,28 ; 0,50$; 0,77 e 0,84 mg.L- ${ }^{1}$. As concentrações de amônia na água foram aferidas utilizando-se a metodologia proposta por Koroleff (1976).

Foi utilizado um delineamento experimental inteiramente casualizado composto por cinco tratamentos e quatro repetições. Os tratamentos foram constituídos pelo uso de água com diferentes concentrações de amônia $(0,06 ; 0,28 ; 0,50 ; 0,77$ e 0,84 mg.L- ${ }^{1}$ ) para ativar os gametas e promover a fertilização artificial, bem como para uso na incubação artificial dos ovos e larvas. As unidades experimentais foram consideradas como: a) uma amostra de sêmen proveniente de um único macho, diluída com água contendo as diferentes concentrações de amônia e, b) $3.800 \pm 105$ ovócitos fertilizados com o sêmen proveniente de cada macho, onde os gametas foram ativados e os ovos incubados em recipientes de volume útil de $2,5 \mathrm{~L}$, com a mesma água contendo as diferentes concentrações de amônia.

Os reprodutores selecionados para uso no experimento foram pesados, identificados e separados por sexo em tanques individuais. As fêmeas foram induzidas com 5,5mg de extrato de hipófise de carpa (EHC)/kg de peso segundo Baldisserotto e Gomes (2010). Os machos receberam uma dose única de 2,5 mg de EHC/kg, juntamente com a segunda dose das fêmeas. Para condução dos procedimentos de indução hormonal, os peixes foram anestesiados em solução de benzocaína a $1 \%$.

Após um período de 181 unidades térmicas acumuladas (UTA), correspondentes a permanência dos reprodutores pelo período de 7 horas em água a $25,7^{\circ} \mathrm{C}$, os peixes foram anestesiados conforme descrito anteriormente e em seguida realizou-se a coleta dos gametas de acordo com Bombardelli et al. (2006). Os gametas (espermatozóides e oócitos) foram coletados a seco, sob leve pressão abdominal no sentido céfalo-caudal. Os primeiros oócitos foram desprezados para evitar possível contaminação por urina ou fezes (BROOKS; TYLER; SUMPTER, 1997), sendo o restante colocados em placa de Petri. A coleta dos gametas masculinos foi idêntica às fêmeas, sendo a primeira gota de sêmen desprezada para evitar possível contaminação (POUPARD et al., 1998) e o restante foi coletado em um tubo de ensaio.

Inicialmente, amostras de sêmen de cada um dos machos foram avaliadas em duplicata para observação dos efeitos da amônia sobre os parâmetros de motilidade espermática. As avaliações dos parâmetros espermáticos foram obtidas pelo método CASA (Computer-Assisted Analasis) segundo Sanches et al. (2010). Para a ativação espermática, empregou-se uma relação de diluição de 1:70 (sêmen:água). Desta mistura $10 \mu \mathrm{L}$ foram colocados em câmara hematimétrica de Neubauer e avaliados em microscópio óptico em objetiva $40 \mathrm{X}$ (Nikon E200). As análises foram realizadas com um segundo de imagem, obtidas $15 \mathrm{~s}$ após o início da ativação espermática. Os parâmetros mensurados foram: motilidade espermática, velocidade espermática curvilínear, velocidade espermática média do caminho, velocidade espermática em linha reta e a linearidade (SANCHES et al., 2010). 
Das fêmeas, foi produzido um "pool" de oócitos para uso nos ensaios de fertilização e incubação artificial dos ovos, os quais foram conduzidos em incubadoras de 2,5L, instaladas em sistemas de recirculação de 250L, separadamente para cada tratamento (BOMBARDELLI et al., 2006; WITECK et al., 2011). A temperatura da água das unidades experimentais foi controlada $\left(25 \pm 1^{\circ} \mathrm{C}\right)$ por meio de aquecimento elétrico.

Para a condução dos procedimentos de fertilização artificial, três mililitros de oócitos (3.800 105 ovócitos) e $0,5 \mathrm{~mL}$ de sêmen foram ativados com $15 \mathrm{~mL}$ de água contendo as diferentes concentrações de amônia. Após a mistura dos gametas e da água, o material foi homogeneizado pelo período de um minuto e, em seguida, transferidos para as incubadoras experimentais. Nove horas após a fertilização artificial, foram mensuradas as taxas de fertilização (AMORIM et al., 2009) por meio da amostragem de 400 ovos de cada unidade experimental (WITECK et al., 2011). A avaliação do percentual de embriões viáveis foi obtida com o auxílio de estereomicroscópio Tecnival SQZ-DS4-TRI35 (10x).

Ainda, seis e 12 horas após a fertilização artificial, foram avaliadas as porcentagens de ovos que estavam em início e término do fechamento do blastóporo. Foi considerado o início, o momento em que aproximadamente $10 \%$ dos embriões apresentavam blastóporo fechado e o término, o momento em que aproximadamente 90\% dos embriões apresentavam blastóporo fechado. Para tanto, seis e 12 horas após a fertilização artificial (RODRIGUES-GALDINO et al., 2009) amostras contendo 20 embriões de cada unidade experimental, foram fixadas em solução de formol tamponado a $4 \%$. O mesmo procedimento foi realizado 16 e 21 horas após a fertilização, para avaliação do percentual de embriões em início e término do desprendimento da cauda. De forma semelhante, foram considerados o momento de início e de termino deste processo quando aproximadamente 10 e $90 \%$ dos embriões estavam com a cauda desprendida, respectivamente. As amostras foram avaliadas em estereoscópio Tecnival SQZ-DS4-TRI35 em aumento de 10X. O período de tempo em termos de UTA, necessário para inicio (IE) e término (TE) da eclosão das larvas foi mensurado. Estes parâmetros foram mensurados por meio de retirada de 40 ovos e/ou larvas de cada tratamento e avaliados em estereoscópio (10x), sendo considerado como tempo para início da eclosão o momento em que $10 \%$ das larvas estavam eclodidos e para o término a eclosão o momento em que $90 \%$ das larvas estavam eclodidas.

Após a eclosão completa das larvas de cada unidade experimental, as mesmas foram fixadas em solução de formol tamponado a 4\%. Estas larvas foram contadas para a estimativa do percentual de deformidade de larvas (JEZIERSKA et al., 2000). Foram avaliadas 1600 larvas de cada tratamento, em estereoscópio (10x).

Os resultados obtidos foram submetidos a análise de variância (ANOVA) a um nível de significância de 5\%. Quando evidenciado efeito dos tratamentos, foi aplicada análise de regressão também a um nível de 5\% de significância. O software utilizado para a realização das análises estatísticas foi o Statistica $7.0^{\circledR}$.

\section{Resultados e Discussão}

Durante o período experimental a temperatura da água permaneceu em $25,7 \pm 1,7^{\circ} \mathrm{C}$, valores considerados adequados para a espécie (GOMES et al., 2000) e os níveis de amônia na água permaneceram inalterados ao longo período experimental $(p=0,96)$. As diferentes concentrações de amônia na água não influenciaram os parâmetros espermáticos do jundiá $(\mathrm{p}>0,05)$, muito embora tenha sido observada uma redução na motilidade espermática e um aumento na velocidade curvilínea quando os níveis de amônia na água aumentaram (Tabela 1). 
Tabela 1. Parâmetros espermáticos do $R$. quelen provenientes de sêmen diluído em água contendo diferentes concentrações de amônia.

\begin{tabular}{lcccccc}
\hline & \multicolumn{7}{c}{ Tratamentos $\left(\mathrm{mg} \mathrm{L}^{-1}\right)$} \\
\cline { 2 - 7 } Variáveis & 0,06 & 0,28 & 0,50 & 0,77 & 0,84 & $\mathrm{p}^{*}$ \\
\hline Motilidade $(\%)$ & 76 & 66 & 66 & 57 & 52 & 0,60 \\
Velocidade curvilínea $\left(\mu \mathrm{m} \mathrm{s}^{-1}\right)$ & 24,72 & 29,72 & 30,26 & 34,89 & 37,12 & 0,73 \\
Velocidade de percurso $\left(\mu \mathrm{m} \mathrm{s}^{-1}\right)$ & 16,96 & 15,94 & 17,92 & 12,26 & 14,16 & 0,74 \\
Velocidade linha reta $\left(\mu \mathrm{m} \mathrm{s}^{-1}\right)$ & 14,41 & 18,63 & 30,40 & 11,69 & 26,50 & 0,57 \\
Linearidade $(\%)$ & 81 & 82 & 82 & 83 & 80 & 0,79 \\
\hline
\end{tabular}

${ }^{*} \mathrm{p}<0,05$ representa diferença significativa entre os tratamentos pelo teste de ANOVA.

Fonte: Elaboração dos autores.

Os valores de motilidade espermática no sêmen diluído com água contendo $0,06 \mathrm{mg} \cdot \mathrm{L}^{-1}$ ficaram próximos aos encontrados em outros estudos desenvolvidos para $R$. quelen. Tessaro et al. (2012) encontraram valores de motilidade espermática variando entre 73 e $84 \%$. Em relação aos tratamentos, somente os maiores níveis de concentração apresentaram valores menores aos observados por Sanches et al. (2010), que verificaram que a motilidade espermática de $R$. quelen foi de $65 \%$. No entanto as velocidades curvilíneas, de percurso e em linha reta obtidas neste estudo ficaram abaixo quando comparado com os valores obtidos pelos autores ora reportados.

Apesar de os níveis de amônia presentes na água não promoverem alterações significativas sobre os parâmetros de motilidade espermática, seus efeitos podem promover danos significativos sobre o processo reprodutivo de peixes. Em geral os espermatozóides de peixes de água doce são imóveis quando ainda estão nos testículos e ganham movimento quando entram em contato com fatores de ativação presentes no meio aquático, a exemplo da pressão osmótica, da presença de íons (INABA, 2008) ou de fatores ovocitários (KINSEY; SHARMA; KINSEY, 2007). Apesar de os mecanismos de ativação e inativação espermática serem pouco compreendidos, sabe-se que em salmonídeos, o início da ativação espermática está relacionado com a redução das concentrações de $\mathrm{K}^{+}$extracelular e o conseqüente o efluxo deste íon intracelular, mediado por canais de iônicos, culminando finalmente na despolarização da membrana celular e na produção de energia para garantir o movimento flagelar (INABA, 2008). Neste caso, a amônia pode interferir no mecanismo de transporte de íons, subtituíndo o $\mathrm{K}^{+}$pelo $\mathrm{NH}_{4}^{+}$ (RANDALL et al., 2000) e conseqüentemente impedir a produção de intermediários metabólicos responsáveis pela ativação espermática (INABA, 2008).

Os níveis de amônia presentes na água também não interferiram sobre as taxas de fertilização dos ovócitos. Resultados semelhantes foram verificados para as taxas de eclosão e para o percentual de larvas normais $(\mathrm{p}>0,05)$ (Tabela 2). O tempo necessário para iniciar e concluir a eclosão das larvas não foi submetido à análise estatística devido a inexistência de repetições, contudo, numericamente seus valores variaram de 682 a 709,1 UTA para o início e de 1085,9 a 1138,5 UTA para o término da eclosão (Tabela 2). 
Tabela 2. Taxas de fertilização de ovócitos, UTA para início e término da eclosão das larvas, parâmetros de desenvolvimento embrionário, taxas de eclosão das larvas e percentual de larvas normais de $R$. quelen provenientes de fertilização e incubação em água com diferentes concentrações de amônia.

\begin{tabular}{lcccccc}
\hline \multirow{2}{*}{ Variáveis } & \multicolumn{7}{c}{ Níveis de amônia na água $(\mathrm{mg} / \mathrm{L})$} \\
\cline { 2 - 7 } & 0,06 & 0,28 & 0,50 & 0,77 & 0,84 & $\mathrm{p}^{*}$ \\
\hline Taxa de fertilização (\%) & 98,36 & 98,86 & 98,49 & 98,70 & 99,19 & 0,58 \\
Início da eclosão (UTA) & 709,1 & 713 & 682 & 688,9 & 682,6 & - \\
Término da eclosão (UTA) & 1128,1 & 1138,5 & 1085,9 & 1095,3 & 1087,1 & - \\
Blastóporo início (\%)* & 9,58 & 12,30 & 58,54 & 43,19 & 95 & 0,00 \\
Blastóporo final (\%) & 93 & 95 & 94 & 97 & 98 & 0,22 \\
Cauda livre início (\%) & 15,97 & 25,13 & 14,26 & 24,19 & 18,93 & 0,84 \\
Cauda livre final (\%) & 64,42 & 81,56 & 45,62 & 67,02 & 61,44 & 0,13 \\
Taxa de eclosão (\%) & 79,98 & 86,08 & 89,03 & 90,01 & 89,52 & 0,51 \\
Normalidade (\%) & 99,91 & 98,80 & 99,89 & 99,85 & 99,87 & 0,45 \\
\hline
\end{tabular}

$\mathrm{UTA}=$ Unidades térmicas acumuladas temporalmente; * Efeito linear $\mathrm{y}=-16,7891+20,1723 * \mathrm{x}$; $* \mathrm{p}<0,05$ representa diferença significativa entre os tratamentos pelo teste de ANOVA.

Fonte: Elaboração dos autores.

Os níveis de contaminação da água com diretamente proporcional $(\mathrm{p}<0,05)$ ao aumento dos amônia interferiram $(\mathrm{p}<0,05)$ no desenvolvimento níveis de amônia na água (Figura 1). Os demais embrionário inicial do $R$. quelen, no início do parâmetros de desenvolvimento embrionário não fechamento do blastóporo (Tabela 2). Neste sofreram influência dos níveis de contaminação da momento ontogenético, ocorreu um comportamento água por amônia ( $\mathrm{p}>0,05)$ (Tabela 2$)$.

Figura 1. Início do fechamento do blastóporo seis horas após a fertilização artificial dos ovócitos e ativação dos gametas de $R$. quelen em água contendo diferentes concentrações de amônia. $\mathrm{p}=\mathrm{p}$-valor.

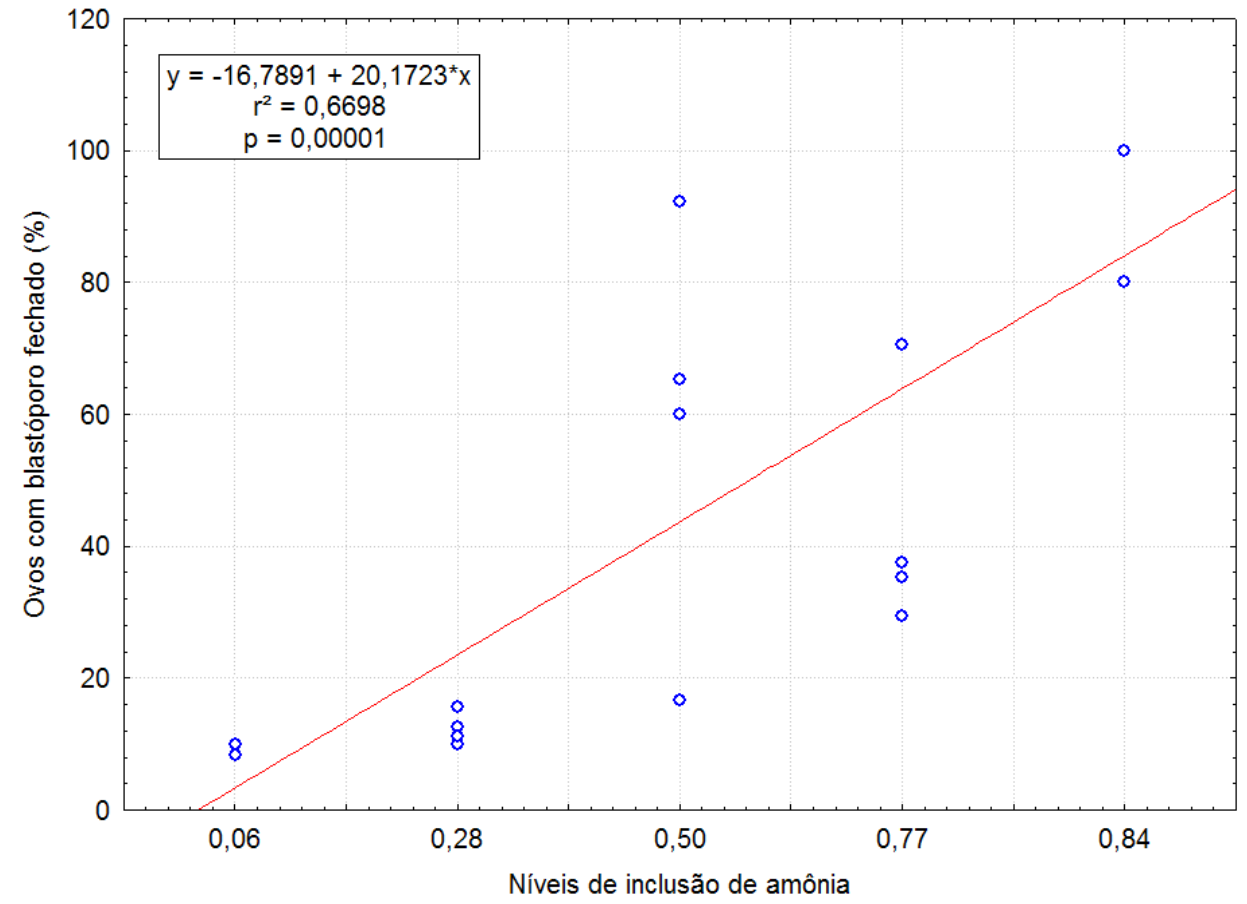

Fonte: Elaboração dos autores. 
Resultados semelhantes em relação à influência da amônia no aumento das taxas de eclosão foram encontrados por Luckenbach et al. (2003) na concentração de $25 \mu \mathrm{g} . \mathrm{L}^{-1}$ de amônia, estudando salmão marrom (Salmo trutta). Segundo os autores, tal fato pode ser explicado pelo fato da amônia acelerar o metabolismo ao interagir nas vias bioquímicas, e desse modo as reservas presentes no vitelo são consumidas mais rapidamente, contribuindo para o desenvolvimento acelerado.

Os resultados do presente experimento podem ser parcialmente corroborados pela hipótese de que, apesar de as membranas biológicas serem permeáveis a água e sais (BALDISSEROTTO, 2002), estas são impermeáveis a alguns íons como o $\mathrm{NH}_{4}^{+}$, para proteger os embriões contra as interferências externas (RANDALL; TSUI, 2002). Contudo, o fato da amônia presente na água ter influenciado apenas o fechamento do blastóporo, pode estar relacionado com a sua absorção em pequenas quantidades durante o processo de ativação do ovócito/ovo, quando ocorre o influxo de água (KINSEY; SHARMA; KINSEY, 2007) e a formação do espaço perivitelino (DEPÊCHE; BILLARD, 1994; GANECO, 2003). Entretanto não se pode descartar a possibilidade de que os níveis de amônia empregados nos tratamentos experimentais podem não terem sido suficientes para induzir danos fisiológicos aos embriões.

Como o experimento foi encerrado logo após a eclosão das larvas, não foi possível avaliar se as concentrações utilizadas nos tratamentos poderiam ter influenciado no desenvolvimento larval. A possibilidade da influência sobre o desenvolvimento larval pode ser sustentada em função da amônia ser facilmente difundida pelo epitélio branquial (RUYET et al., 1997) e desse modo, seus principais efeitos fisiológicos estão relacionados com prejuízos metabólicos relacionados com a redução do crescimento, com danos provocados ao epitélio branquial que promove dificuldades nas trocas gasosas e ao sistema de osmorregulação (BOLNER, 2007). Em geral, níveis de amônia entre 0,06 e 3,1 mg.L $\mathrm{L}^{-1}$ interferem no desenvolvimento e sobrevivência de peixes (ABDALLA; MACNABB, 1998; FRANCES; NOWAK; ALLAN, 2000; WICKS; RANDALL, 2002; FOSS et al., 2003; EL-SHAFAI et al., 2004; LAMARIÉ et al.., 2004). Embora esses valores possam variar de acordo com a idade, espécie e tempo de exposição, juvenis de $R$. quelen, toleram níveis de amônia entre 0,01 até $1,86 \mathrm{mg} . \mathrm{L}^{-1}$ sem a ocorrência de prejuízos aparente (WEISS; ZANIBONI-FILHO, 2009; MIRON et al., 2011).

Embora não tenham sido encontrados efeitos significativos da inclusão de amônia na água sobre a maioria dos parâmetros avaliados na fertilização e no desenvolvimento de larvas de $R$. quelen, não existem trabalhos que descrevam o efeito da amônia nessas fases. Assim, são necessárias novas pesquisas neste campo para elucidar os efeitos da amônia sobre a fertilização artificial e o desenvolvimento inicial das espécies em sistemas de criação artificial, especialmente porque a crescente preocupação com o uso da água está levando ao emprego de sistemas de criação com filtragem e recirculação da água, onde a amônia pode ser um fator limitante.

Conclui-se que a fertilização de oócitos de $R$. quelen em água contendo até $0,84 \mathrm{mg}$ de amônia total/L de água acelera o desenvolvimento inicial mas não interfere nos parâmetros espermáticos, nas taxas de fertilização ou eclosão das larvas, bem como nas larvas classificadas como normal.

\section{Referências}

ABDAlLA, A. A.; MACNABB, C. D. Acute and sublethal growth effects of um-ionized ammonia to nile tilapia Oreochromis niloticus. The Progressive FishCulturist, Baltimore, v. 58, n. 1, p. 117-123. 1998.

AMORIM, M. P.; GOMES, B. V. C.; MARTINS, Y. S; SATO, Y; RIZZO, E.; BAZZOLI, N. Early development of the silver catfish Rhamdia quelen (Quoy \& Gaimard, 1824) (Pisces:Heptapteridae) from the São Francisco River Basin, Brazil. Aquaculture Research, Oxford, v. 40, n. 2, p. 172-180, 2009.

BALDISSEROTTO, B. Fisiologia de peixes aplicada à piscicultura. Santa Maria: UFSM, 2002. 212 p. 
BALDISSEROTTO, B.; GOMES, L. C. Espécies nativas para a piscicultura no Brasil. 2. ed. Santa Maria: UFSM, 2010. $608 \mathrm{p}$.

BALDISSEROTTO, B.; RADÜNZ NETO, J. Criação de jundiá. Santa Maria: UFSM, 2004. 232 p.

BECKER, A. G.; GARCIA, L. O.; KOCHHANN, D.; GONÇALVES, J. F.; LORO, V. L.; BALDISSEROTTO, B. Dissolved oxygen and ammonia levels in water that affect plasma ionic content and gallbladder bile in silver catfish. Ciência Rural, Santa Maria, v. 39, n. 6, p. 17681773, 2009.

BOLNER, K. C. S. Parâmetros metabólicos e ions plasmáticos de piavas (Leporinus obtusidens) expostas a diferentes níveis de oxigênio dissolvido e amônia. 2007. Dissertação (Mestrado em Biologia) - Curso de Pós Graduação em Biodiversidade Animal. Universidade Federal Santa Maria, Santa Maria.

BOMBARDELLI, R. A.; MÖRSCHBÄCHER, E. F.; CAMPAGNOLO, R.; SANCHES, E. A.; SYPERRECK, M. A. Dose inseminante para fertilização artificial de ovócitos de jundiá cinza, Rhamdia quelen (Quoy \& Gaimard, 1824). Revista Brasileira de Zootecnia, Viçosa, MG, v. 35, n. 4, p. 1251-1257, 2006.

BROOKS, S.; TYLER, C. R.; SUMPTER, J. P. Egg quality in fish: what makes a good egg? Reviews in Fish Biology and Fisheries, New York, v. 7, n. 4, p. 387-416, 1997.

DEPÊCHE, J.; BILLARD, R. Embryology in fish: a review. Paris: Société Française d'Ichtyologie, 1994. $123 \mathrm{p}$.

EL-SHAFAI, S. A.; EL GOHARY, F. A.; NASR, F. A.; STEEN, N. P. V.; GIJZEN, H. J. Chronic ammonia toxicity to duckeed-fed tilapia (Oreochromis niloticus). Aquaculture, Amsterdam, v. 232, n. 1, p. 117-127, 2004.

FAO. The State of world fisheries and aquaculture. Roma: Food and Agriculture Organization of United Nations, 2010.

FOSS, A.; EVENSEN, T. H.; VOLLEM, T.; OIESTAD, V. Effects of chronic ammonia exposure on growth and food conversion efficiency in juvenile spotted wolffish. Aquaculture, Amsterdam, v. 228, n. 1, p. 215-224, 2003.

FRANCES, J.; NOWAK, B. F.; ALLAN, G. L. Effects of ammonia on juvenile silver perch (Bidyanus bidyanus). Aquaculture, Amsterdam, v. 183, n. 1, p. 95-103, 2000.

GANECO, L. M. Análise dos ovos de piracabjuba, Brycon orbignyanus (Valenciennes, 1849), durante a fertilização e o desenvolvimento embrionário, sob condições de reprodução induzida. 2003. Dissertação (Mestrado em Aquicultura) - Curso de Pós-Graduação em Aquicultura, Universidade Estadual Paulista Julio Mesquita Filho, Jaboticabal.

GOMES, L. C.; GOLOMBIESKI, J. I.; GOMES, A. R. C.; BALDISSEROTTO, B. Biologia do Jundiá Rhamdia quelen (Teleostei, Pimelodidae) Ciência Rural, Santa Maria, v. 30, n. 1, p. 179-185, 2000.

HILBIG, C. C.; BOMBARDELLI, R. A.; SANCHES, E. A.; OLIVEIRA, J. D. S.; BAGGIO, D. S.; SOUZA, B. E. Efeito do chumbo sobre a fertilização artificial e incubação de ovos de jundiá cinza (Rhamdia quelen). Acta Scientiarum Animal Sciences, Maringá, v. 30, n. 2, p. 217-224, 2008.

INABA, K. Molecular mechanisms of the activation of flagellar motility in sperm. In: ALAVI, S. M. H.; COSSON, J.; COWARD, K.; RAFIEE, G. (Ed.). Fish spermatology. Oxford: Alpha Science International Ltd, 2008, p. 267-280.

JEZIERSKA, B.; LUGOWSKA, K.; WITESKA, M.; SAMOWSKI, P. Malformations of newly hatched common carp larvae. Electronic Journal of Polish Agricultural Universities, Poznan, v. 2, n. 2, p. 1-10, 2000.

KINSEY, W. H.; SHARMA, D. E.; KINSEY, S. C. Fertilization and egg activation in fishes. In: BABIN, P.; CERDÀ, J. E.; LUBENZ, E. (Ed.). The fish oocyte: from basic studies to biotechnologycal applications. Springer: Dordrecht, 2007. p. 397-409.

KOROLEFF, F. Determination of ammonia. In: GRASSHOFF, K. (Ed.) Methods of seawater analysis. Verlag Chemi: Weinheim, 1976. 317 p.

KÜTTER, M. T.; BEMVENUTI, M. A.; MORESCO, A. Feeding strategy of the jundiá Rhamdia quelen (Siluriformes, Heptapteridae) in costal lagoons of southern Brazil. Acta Scientiarum Biological Sciences, Maringá, v. 31, n. 1, p. 41-47, 2009.

LAMARIÉ, G.; DOSDAT, A.; COVÈS, D.; DUTTO, G.; GASSET, E.; RUYET, P. Effect of chronic ammonia exposure on growth of European seabass (Dicentrarchus labrax) juveniles. Aquaculture, Amsterdam, v. 229, n. 1, p. 479-491, 2004.

LUCKENBACH, T.; FERLING, H.; GERNHÖFER, M.; KÖHLER, H.; NEGELE, R.; PFEFFERLE, E.; TRIEBSKORN, R. Developmental and subcellular effects of chronic exposure to sub-lethal concentrations of ammonia, PAH and PCP mixtures in brown trout (Salmo trutta f. fario L.) early life stages. Aquatic Toxicology, Oxford, v. 65, n. 1, p. 39-54, 2003.

MAFFEZZOLLI, G.; NUÑER, A. P. O. Crescimento de alevinos de jundiá, Rhamdia quelen (Pisces, Pimelodidae), em diferentes concentrações de oxigênio dissolvido. Acta 
Scientiarum Biological Science, Maringá, v. 28, n. 1, p. 41-45, 2006.

MIRON, D. S.; MORAES, B.; BECKER, A. G.; CRESTANI, M.; SPANEVELLO, R, LORO, V. L.; BALDISSEROTTO, B. Ammonia and $\mathrm{pH}$ effects on some metabolic parameters and gill histology of silver catfish, Rhamdia quelen (Heptapteridae). Aquaculture, Amsterdam, v. 277, n. 3, p. 192-196, 2008.

MIRON, D. S.; BECKER, A. G.; LORO, V. L.; BALDISSEROTO, B. Waterborne ammonia and silver catfish, Rhamdia quelen: survival and growth. Ciência Rural, Santa Maria, v. 41, n. 2, p.349-353, 2011.

MPA. Boletim Estatístico da Pesca. Ministério da Pesca e Aquicultura, Brasília, 2009.

OSTRENSKY, A.; BOEGER, W. A. Piscicultura fundamentos e técnicas de manejo. Guaíba: Agropecuária, 1998. $211 \mathrm{p}$.

PERDICES, A.; BERMINGHAM, E; MONTILLA, A.; DOADRIO, I. Evolutionary history of the genus Rhamdia (Teleostei: Pimelodidae) in Central America. Molecular Phylogenetics and Evolution, Waltham, v. 25, n. 1, p. 172-189, 2002.

PEREIRA, L. P. F.; MERCANTE, C. T. J. A amônia nos sistemas de criação de peixes e seus efeitos sobre a qualidade da água. Uma revisão. Boletim do Instituto de Pesca, São Paulo, v. 31, n. 1, p. 81-88, 2005.

POUPARD, G. P.; PAXION, C.; COSSON, J.; JEULIN, C.; FIERVILlE, F.; BILLARD, B. Initiation of carp spermatozoa motility and early ATP reduction after milt contamination by urine. Aquaculture, Amsterdam, v. 160, n. 3, p. 317-328, 1998.

QUOY, J. R. C.; GAIMARD, J. P. Zoologie. In: FREYCINET, L. D. (Ed.). Voyage autour du monde. Paris: Chez Pillet Aine, 1824.

RAABE, W.; LIN, S. Pathophysiology of ammonia intoxication. Experimental Neurology, Michigan, v. 87, n. 3, p. 519-532, 1985.

RANDALL, D. J.; TSUI, T. K. N. Ammonia toxicity in fish. Marine Pollution Bulletin, Amsterdam, v. 45, n. 1, p. 17-23, 2002.

RANDALL, D.; BURGGREN, W.; FRENCH, K.; FERNALD, R. ECKERT Fisiologia animal: mecanismos e adaptações. Rio de Janeiro: Guanabara Koogan, 2000. 729 p.

RODRIGUES-GALDINO, A. M.; MAIOLINO, C. V.; FORGATI, M.; DONATTI, L.; MIKOS, J. D.; CARNEIRO, P. C. F.; RIOS, F. S. Development of the neotropical catfish Rhamdia quelen (Siluriformes,
Heptapteridae) incubated in different temperature regimes. Zygote, Cambridge, v. 18, n. 2, p. 131-144, 2009.

RUYET, J. P.; GALLAND, R.; LE ROUX, A.; CHARTOIS, H. Chronic ammonia toxicity in juvenile turbot (Scophthalmus maximus). Aquaculture, Amsterdam, v. 154, n. 2, p. 155-171, 1997.

SANCHES, E. A.; BOMBARDELLI, R. A.; MARCOS, R. M.; NEUMANN, G.; TOLEDO, C. P. R.; ROMAGOSA, E. Sperm motility of Rhamdia quelen studied using computer-assisted analysis by open-source software. Aquaculture Research, Oxford, v. 42, n. 1, p. 1-4, 2010.

SANCHES, E. A.; NEUMANN, G.; BAGGIO, D. M.; BOMBARDELLI, R. A.; PIANA, P. A.; ROMAGOSA, E. Time and temperature on the storage of oocytes from jundiá catfish, Rhamdia quelen. Aquaculture, Amsterdam, v. 319, n. 3, p. 453-458, 2011.

SILVA, G. S.; JARDIM, W. F. Um novo índice de qualidade das águas para proteção da vida aquática aplicado ao Rio Atibaia, região de Campinas/Paulínia SP. Química Nova, São Paulo, v. 29, n. 4, p. 689-694, 2006.

TESSARO, T.; TOLEDO, C. P. R.; NEUMANN, G.; KRAUSE, R. A.; MEURER, F.; NATALI, M. R. M.; BOMBARDELLI, R. A. Growth and reproductive characteristics of Rhamdia quelen males fed on different digestible energy levels in the reproductive phase. Aquaculture, Amsterdam, v. 326-329, n. 1, p. 74-80, 2012.

WANG, Y.; WALSH, P. J. High ammonia tolerance in fishes of the family Batrachoididae (Toadfish and Midshipmen). Aquatic Toxicology, Maryland Heights, v. 50, n. 3, p. 205-219, 2000.

WEISS, L. A.; ZANIBONI-FILHO, E. Survival of diploid and triploid Rhamdia quelen juveniles in different ammonia concentrations. Aquaculture, Amsterdam, v. 298, n. 3, p. 153-156, 2009.

WICKS, B. J.; RANDALL, D. J. The effect of sub-lethal ammonia exposure on fed and unfed rainbow trout: the role of glutamine in regulation ammonia. Comparative Biochemistry and Physiology, Amsterdam, v. 132, n. 2, p. 275-285, 2002.

WITECK, L.; BOMBARDELLI, R. A.; SANCHES, E. A.; OLIVEIRA, J. D. S.; BAGGIO, D. M; SOUZA, B. E. Motilidade espermática, fertilização dos ovócitos e eclosão dos ovos de jundiá em água contaminada por cádmio. Revista Brasileira de Zootecnia, Viçosa, MG, v. 40, n. 3, p. 477-481, 2011. 
